\title{
Stochastic semi-Lagrangian micro-macro calculations of liquid crystalline solutions in complex flows
}

\author{
Juan Luis Prieto ${ }^{a, d, *}$, Patrick Ilgc, Rodolfo Bermejo ${ }^{d}$, Manuel Laso ${ }^{a, b}$ \\ anstitute for Optoelectronics and Microsystems (ISOM), Universidad Politécnica de Madrid, Cindad Universitaria, 28040 Madrid, Spain \\ b Department of Chemical Engineering, ETSI, Universidad Politécnica de Madrid, jose Gutlertez Abascat 2, 28006 Madrid, Spain \\ 'Department of Materials, ETH Ztirich, Polymer Physics, Wolfgang-Pauli-Strasse 10, $\mathrm{CH}-8093$ Zatrich, Switzerland \\ d Department of Applied Mathematics, ETSI, Universidad Politécnica de Madrid, josé Gutlértez Abascal 2, 28006 Madrid, Spain
}

Keywords:

Liquid crystal

Stochastic simulation

Semi-Lagrangian scheme

Micro-macio

Finite element

Richatdson Eulet-Matuyama

\begin{abstract}
A B S T R A C T
A general method for the simulation of complex flows of liquid crystalline polymers ( $\mathrm{LCP}$ s) using a stochastic semi-Lagrangian micro-macro method is introduced. The macroscopic part uses a spatialtemporal second order accurate semi-Lagrangian algorithm, where ideas from the finite element and natural element methods are mixed in order to compute average quantities. The microscopic part employs a stochastic interpretation of the Doi-Hess LCP model, which is discretized with a second order Richardson extrapolated Euler-Maruyama scheme.

The new method is validated and tested using the benchmark problem of flow between rotating eccentric cylinders. In a decoupled analysis, a discussion on the sensibility of the scalar order parameter to the macroscopic flow is offered. For the coupled situation, the proposed method predicts disclinations at certain regions of the geometry, as well as an accentuated abatement of the flow as the strength of the micro-macro interaction increases. Further examples are provided at different Peclet and concentration numbers to gain insight on the behavior of complex flows of LCPS in the eccentric cylinder geometry.

The generality and robustness of the method, as well as its accurate prediction of LCP behavior under complex flows are main features of the implementation.
\end{abstract}

\section{Introduction}

Liquid crystals (LCS) are anisotropic fluids in which the orientation of the rigid molecules they comprise are affected by the history of the flow; all the more so since most of the properties of the LC will greatly depend on the fluctuations of the unit vector field (the so-called directors $\mathbf{d}$ ) which represents the different average orientation of the particles. Furthermore, the actual coupling between flow and particle orientation plays in both directions, according to rules not yet ascertained. Typically, LC molecules (like the extensively studied pentyl-cyano-biphenyl, or 5CB molecule) possess moderately high aspect ratios, and liquid crystalline polymers (LCPs) have rigid units that can behave as rodlike particles, with extremely high aspect ratios.

Along with the Leslie-Ericksen theory [1-3] and the Landaude Gennes model (see, e.g. [4]), the Hess-Doi model $([5,6]$ ) has been widely employed to simulate complex flows of LCPS. In the

\footnotetext{
+ Corresponding author: Tel:: +34 913364261: tax: +34676225060.

E-mail addresses: juanluis,prieto@upm,es (J.L. Ptieto). pilg@mat.ethz.ch (P. IIg), Dermejo@etsii.upm.es (R. Bermejo), mlaso@etsii.upm.es (M. Laso).
}

Hess-Doi model, the LCP is envisioned as an ensemble of particles with infinite aspect ratio (rigid rods) that undergo Brownian motion and interact via a self-consistent mean-field potential. However, the complexity of the problem demands further simplifications, like reducing the dimensions of the configuration space or employing simplified expressions for the rotary diffusivity and the mean-field potential.

Using the Hess-Doi model, remarkable insight has been gained on the behavior of LCPs in pure shear homogeneous flows [7-18]. Poiseuille flow (see, e.g. [19,20]) and pressure-driven flows [21]; however, complex flows have received comparatively little attention, possibly due to the computational cost of accurately reflecting the historical effects and the flow modification induced by the soaltered particle orientations. An exception is the work by Feng and Leal [22], who investigated the start-up of LCPs according to the Hess-Doi model in an eccentric cylinder geometry.

In order to avoid a direct simulation of the distribution function appearing in the Hess-Doi kinetic model, it is a common approach to combine the macroscopic and the statistical models in some form of closure approximation [9-11,16,18]. Since closure approximations can severely alter the time evolution of the alignment tensor, a wrong choice could lead to inconsistencies with the kinematic model they were based on [23], or produce macroscopic results 
qualitatively different from those predicted by the distribution function. In this respect, the recent effort by Kröger and co-workers [24] should definitely help in the election of a statistically consistent closure approximation. An investigation of appropriate closure approximations and their possible qualitative impact on the results in the eccentric cylinder geometry can be found in $[25,26]$. As an alternative, hybrid methods propose a simultaneous resolution of both the macroscopic equations (Navier-Stokes equations with a source term due to the polymer) and the diffusion equations for the particle orientations.

Thus, the two main families of methods used nowadays attempt either to solve the diffusion equation in configuration space (Fokker-Planck), or to couple discretization techniques with a direct solution of the associated stochastic differential equation for a large ensemble of trajectories. The direct solution of the Fokker-Planck equation is arguably superior in terms of convergence for small numbers of degrees of freedom in homogeneous fields, if the proper trial functions are chosen; spherical harmonics have been traditionally employed [7], but recently. Suen et al. [27] presented the wavelet expansion as a sound alternative. As to the second class of methods, explored in [28-30], they are still rather competitive when complex flows and precise, theological information come into scene (see, e.g. the review by Keunings on micro-macro methods for viscoelastic fluids for a more detailed exposition [31]).

Following this latter approach, we intend to devise an efficient and accurate micro-macro method to simulate complex flows of LCPS, with a focus on the solution of the fully coupled problem. and not so much about how the LCP reacts to given velocity fields. The purpose is threefold: (1) to introduce a general method for the simulation of LCPs, assessing its capabilities and drawbacks in the context of complex LCPs flows; (2) to address the discrepancies or concordances between the values predicted by this method and other solution methods such as closure approximations or the spherical harmonics technique for the rheological variables of interest, namely, the scalar order parameter $S$ or the director vector d; and (3) to study the effect of the LCP over a macroscopic, quasi-Newtonian Stokes flow.

The present paper is organized as follows. Section 1 serves as an introduction to the topic of LCPS and their simulation. The mathematical description of the problem is exposed in Section 2. Section 3 provides numerical details on the algorithm, including a succinct description of the micro-macro method used. In Section 4 we show results for the eccentric cylinder geometry, comparing them with those of previous works and discussing the discrepancies that might arise. Finally, Section 5 summarizes the main objectives and conclusions of this work

\section{Mathematical formulation}

The description of the LCP solution will follow the Hess-Doi model $[5,6]$, where the LCP solution is thought of as an ensemble of neutrally buoyant rodlike particles with infinite aspect ratio. The LCP solution is subject to a macroscopic hydrodynamic flow which depends on the problem geometry. Conversely, the particle orientations, measured by the director vectors, exert a certain stress back on the flow which translates into a flow modification, should the concentration of the polymer in the solution be high enough.

\subsection{Macroscopic equations: dimensionless form}

We consider the flow of a constant density LCP solution in a bounded domain $D \subset \mathbb{R}^{2}$ with boundary $\Gamma$ during a time interval $[0, T]$. The boundary $\Gamma$ is composed of various pieces $\Gamma^{i}, \Gamma^{s}, \Gamma^{o}$ denoting inflow, solid and outflow boundaries, respectively. The governing equations, assuming there are no body forces acting on the flow, are the following:

$\left\{\begin{array}{l}\rho \frac{D v}{D t}+\nabla p=\eta_{s} \Delta \mathbf{v}+\nabla \cdot \tau_{\mathrm{LC}} \text { in } D \times(0, T], \\ \nabla \cdot \mathbf{v}=0 \text { in } D \times(0, T] .\end{array}\right.$

These equations are to be solved with the initial conditions

$\mathbf{v}(\mathbf{x}, 0)=\mathbf{v}_{0}(\mathbf{x}) \forall \mathbf{x} \in D$,

and the boundary conditions

$$
\begin{aligned}
& \mathbf{v}(\mathbf{x}, t)=\mathbf{0} \text { on } \Gamma^{s} \forall t, \\
& -\mathbf{p n}+\eta_{s} \frac{\partial \mathbf{v}}{\partial \mathbf{n}}=\mathbf{g}(\mathbf{x}, t) \text { on } \Gamma^{o} \forall t, \\
& -\mathbf{n} \cdot \mathbf{v}(\mathbf{x}, t)=\boldsymbol{a}(\mathbf{x}, t) \text { on } \Gamma^{i} \forall t .
\end{aligned}
$$

Неге, $v$ and $p$ denote the flow velocity and the hydrodynamic pressure, respectively: $\rho$ is the constant density of the solution; $\mathbf{n}$ is the unitary outward normal vector at the boundary; $g$ and $a$ are known data: $(D / D t) \equiv(\partial / \partial t)+\mathbf{v} \cdot \nabla$ represents the material derivative operator; the total stress tensor $\boldsymbol{\tau}$ is defined as $\boldsymbol{\tau}=\boldsymbol{\tau}_{\mathrm{S}}+\boldsymbol{\tau}_{\mathrm{LC}}$, where the Newtonian stress tensor is $\boldsymbol{\tau}_{S}=\eta_{S}\left(\nabla \mathbf{v}+(\nabla \mathbf{v})^{T}\right)$ with $\eta_{s}$ being the solvent viscosity: and $\tau_{\mathrm{Lc}}$ is the liquid crystal contribution due to anisotropic particle orientations.

For a circular geometry such as the eccentric cylinder device (see Fig. 3), one can select a characteristic length $L$, a characteristic time $T$, and express the rest of macroscopic magnitudes in the following manner: $\mathbf{v}^{*}=\mathbf{v} /(\omega \mathrm{L})$ as the dimensionless velocity, $p^{*}=p /\left(\eta_{s}(t)\right)$ as the dimensionless pressure, and $\tau_{\mathrm{LC}}^{*}=\tau_{\mathrm{LC}} /\left(3 n k_{\mathrm{B}} \Theta\right)$ as the stress tensor in non-dimensional form. Неге, ${ }^{* *}$ denotes a dimensionless magnitude, $n$ is the number density of rodlike particles, $k_{B}$ is the Boltzmann constant, $\omega$ is the angular frequency, and $\Theta$ the absolute temperature. Then, one can write the dimensionless equivalent of Eqs. (1)-(3):

$\left\{\begin{array}{l}\operatorname{Re} \frac{D \mathbf{v}^{+}}{D t}+\nabla p^{*}=\Delta \mathbf{v}^{*}+\frac{c}{P e} \nabla \cdot \tau_{\mathrm{LC}}^{*} \text { in } D^{*} \times\left(0, T^{*}\right], \\ \nabla \cdot \mathbf{v}^{*}=0 \text { in } D^{+} \times\left(0, T^{*}\right] .\end{array}\right.$

$\mathbf{v}^{*}\left(\mathbf{X}^{*}, 0\right)=\frac{\mathbf{v}_{0}(\mathbf{X})}{\omega L} \equiv \mathbf{v}_{0}^{*}(x) \forall \mathbf{X}^{*} \in D^{*}$.

$\mathbf{v}^{*}\left(\mathbf{x}^{+}, t^{+}\right)=\mathbf{0}$ on $\Gamma^{s *} \forall t^{+}$

$-p^{*} \mathbf{n}^{*}+\frac{\partial \mathbf{v}^{*}}{\partial \mathbf{n}^{*}}=\frac{\mathbf{g}(\mathbf{x}, t)}{\eta_{S} \omega}=\mathbf{g}^{*}$ on $\Gamma^{0 *} \forall t^{*}$,

$-\mathbf{n}^{*} \cdot \mathbf{v}^{*}\left(\mathbf{x}^{*}, t\right)=\frac{a(\mathbf{X}, t)}{\omega L} \equiv a^{*}$ on $\Gamma^{i *} \forall t^{*}$,

where $D_{r}$ is the rotary diffusivity of the LCP particles; $R e=p \omega \mathrm{L}^{2} / \eta_{s}$ is the Reynolds number; $c=n k_{B} \Theta /\left(2 \eta_{s} D_{r}\right)$ is the concentration parameter; and $\mathrm{Pe}=\omega /\left(6 \mathrm{D}_{\mathrm{r}}\right)$ is the Peclet number.

\subsection{Microscopic equations: dimensionless form}

The description of the LCP according to the Hess-Doi model $[7,32,33]$ for rodlike particles with aspect ratio $\rightarrow \infty$, prescribes an evolution equation for the particle orientation probability density function $\psi(\mathbf{x}, t, \mathbf{u})$ of the form:

$$
\begin{aligned}
& \frac{D \psi}{D t}+\frac{\partial}{\partial \mathbf{u}} \cdot[(\mathbf{u} \cdot \nabla \mathbf{v}-\mathbf{u u u u}: \nabla \mathbf{v}) \psi] \\
& -D_{r} \frac{\partial}{\partial \mathbf{u}} \cdot\left\{\left[\frac{\partial \psi}{\partial \mathbf{u}}+\psi r \frac{\partial}{\partial \mathbf{u}}\left(\frac{V}{k_{B} \Theta}\right)\right]\right\}=0,
\end{aligned}
$$

where $f(\mathbf{x}, t, \mathbf{u})$ is the probability density that a rodlike particle aligns itself according to the unit vector $\mathbf{u}$ at time $t$ and position $\mathbf{x}: \nabla \mathbf{v}$ is the velocity gradient; $V$ is the mean-field potential; and $D_{\text {, }}$ 
is the rotary diffusivity for a rodlike particle in an isotropic solution. The stochastic equation equivalent to $\mathrm{Eq}$. (7) is given by $[34,7,30]$ :

$$
\begin{aligned}
d \mathbf{u}= & (\mathbf{I}-\mathbf{u} \mathbf{u}) \cdot\left\{\left[\mathbf{u} \cdot \nabla \mathbf{v}-D_{r} \frac{\partial}{\partial \mathbf{u}}\left(\frac{V}{k_{\mathrm{B}} \Theta}\right)\right] d t+\sqrt{2 D_{r}} d \mathbf{W}\right\} \\
& -2 D_{r} \mathbf{u} d t
\end{aligned}
$$

with I being the identity tensor and $\mathbf{W}(\mathbf{t}) \equiv\left\{W_{1}(t), W_{2}(t), W_{3}(t)\right\}$ a vector whose components are independent Wiener processes. We here choose the Maier-Saupe form [35,6] of the mean-field potential:

$V=V^{M S}(\mathbf{u})=-\frac{3}{2} U k_{B} \Theta \mathbf{u u}:\{\mathbf{u} \mathbf{u}\}$,

where $U$ is the dimensionless strength of the $L C$-interaction, and $(\cdot)$ indicates an orientational ensemble average.

Remark 1. The mean-field interaction strength $U$ and rotary diffusivity $D_{r}$ depend on the concentration of LCP molecules. Following previous approaches $[21-23,25,26]$, we consider situations where the orientations of the LCPS molecules can vary within the domain but the concentration is spatially homogeneous. We note that the method proposed here can be applied also to the more general situation of inhomogeneous concentrations (see, e.g. [36-40]); however, we focus on the case of homogeneous concentrations in order to facilitate comparison of our new method to previous results.

Considering a circular geometry with the same characteristic length $L$ and characteristic time $T$ as in Section (2.1), we can write the following dimensionless variables: $\mathbf{K}^{*}=\mathbf{k} T \equiv\left(\nabla \mathbf{v}^{*}\right)^{T}$ as the transpose of the dimensionless velocity gradient and $D_{\tau}^{*}=D_{\Gamma} T$ as the dimensionless rotary diffusivity, which relates to the Peclet number according to $\mathrm{Pe} \equiv 1 /\left(6 D_{r}^{*}\right)$. Thus, considering that $d \mathbf{W}^{*}$ has dimensions of $\sqrt{t^{4}}$ and the mean field potential given by Eqs. (8) and (9) can be rewritten as:

$$
\begin{aligned}
d \mathbf{u}= & (\mathbf{I}-\mathbf{u u}) \cdot\left[\left(\kappa^{*} \cdot \mathbf{u}+\frac{U}{2 P e}\langle\mathbf{u} \mathbf{u}\rangle \cdot \mathbf{u}\right) d t^{*}+\sqrt{\frac{1}{3 P e}} d \mathbf{W}^{*}\right] \\
& -\frac{\mathbf{u}}{3 P e} d t^{*} .
\end{aligned}
$$

The initial distribution $\mathbf{u}(t=0)$ should be set according to the conditions specified in the problem, e.g. perfect alignment with the ' $\boldsymbol{x}$-axis' $\Rightarrow \mathbf{u}(t=0)=\mathbf{e} \boldsymbol{x}$, where $\mathbf{e} \_\boldsymbol{x}$ represents the unit vector along that axis; or an isotropic condition (perfect disorder).

The dimensionless contribution of the polymer to the stress tensor is obtained by $[6,30,25]$ :

$\boldsymbol{\tau}_{\mathrm{LC}}^{*}=\mathbf{S}-U[\mathbf{S} \cdot\langle\mathbf{u u}\rangle-\mathbf{S}:\langle\mathbf{u u u u}\rangle]+\frac{\beta P e}{\left(n L_{p}^{3}\right)^{2}} \kappa^{* T}:\langle\mathbf{u u u u}\rangle$

where $\mathbf{S}$ is the alignment tensor, defined as $\mathbf{S}=\langle\mathbf{u u}\rangle-\mathbf{I} / 3, \beta=$ $O\left(10^{3}\right)$ is an empirical parameter determining the strength of the viscous stress contribution, and $L_{p}$ is the length of the rodlike particles. For concentrated solutions that are considered here, often $\beta=0$ is chosen since the viscous stress contribution is assumed to be negligible [6]. We keep this term so as to compare our results to previous studies.

\section{Numerical formulation}

To integrate Eqs. (4)-(6) we employ the semi-Lagrangian finite element method introduced in [41]. The main features of this method are the following. (1) The time discretization is performed by a second order in time Backward-Difference-Formula (BDF2)

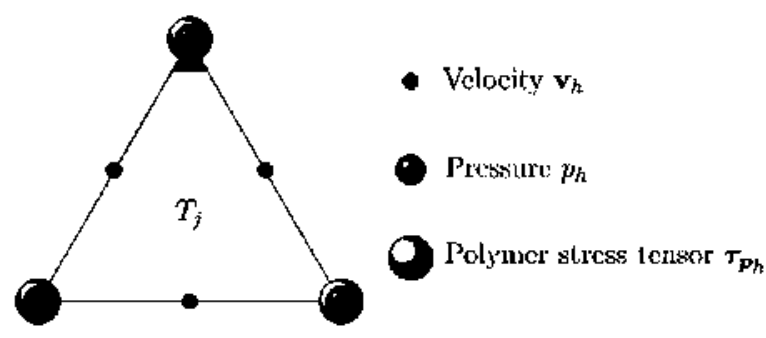

Fig. 1. Extended Taylor-Hood computational element, $P_{2} / P_{1} / P_{1}$ for velocity, pressure and polymer stress tensor nodes.

along the characteristics curves of the material derivative operator $(D v / D t)$. (2) The use of $P_{2} / P_{1}$ Taylor-Hood element for space discretization of velocity and pressure (Fig. 1).

We must note that this element satisfies the so-called inf-sup condition. (3) The use of $P_{1}$-element for the space discretization of tensorial quantities. Specifically, the finite element space for tensor fields $\boldsymbol{S}_{\sqrt{1}}$ of order $r \geq 2$ in $\mathbb{R}^{2}$ is defined as

$S_{h}^{(r)}=\left\{S_{n_{1}} \in C^{0}(\bar{D})^{2^{r}}:\left.S_{h_{1}}\right|_{r_{j}}=P_{1}\left(T_{j}\right)^{2^{r}} .1 \leq j \leq N E\right\}$,

where $N E$ is the total number of triangles of the finite element mesh, and $T_{j}$ denotes the $j$ th triangle. Here, $C^{0}(\bar{D})^{2^{r}}$ means that each component of the tensor $\boldsymbol{S}_{h}$ of order $r$ is a continuous and bounded function defined on $\bar{D}$. Hence, each $S_{h} \in S_{h}^{(r)}$ is then expressed as

$\mathbf{S}_{h}(\mathbf{x})=\sum_{k=1}^{M P}\left(S_{i_{1}} \ldots i_{r}\right){ }_{k} \Psi_{k}(\mathbf{x}), 1 \leq i_{1}, \ldots, i_{r} \leq 2$

where $\left(S_{i_{1} \ldots i_{r}}\right)_{k}=S_{h}\left(\mathbf{x}_{k}\right)$ are the values of $\boldsymbol{S}_{h}(\mathbf{x})$ at the vertices $\mathbf{x}_{k}$ of the triangles, $M P$ the number of pressure nodes, $M V$ the number of velocity nodes, and $\left\{\Psi_{k}\right\}$ denote the set of global basis functions of the subspace of piecewise linear polynomials defined on $\bar{D}$. Since the difference of the numerical method of this paper with respect to the one of [41] lies in the calculation of the solution of the SDE Eq. (10), we proceed now to describe it.

\subsection{Numerical calculation of the SDE Eq. (10)}

To approximate the solution of the SDE (Eq. (10)) we employ the adaptive algorithm of [41] to calculate the position of the the center of mass $\mathbf{X}^{n}$ of each rodlike particle at time instant $t_{n}$; whereas $\mathbf{u}^{\mathrm{n}}$ is the numerical solution at time $t_{n_{1}}$ which is obtained by applying Richardson extrapolation to the solutions $\tilde{\mathbf{u}}_{\Delta t / 2}^{n}$ and $\tilde{\mathbf{u}}_{\Delta t}^{\mathrm{n}}$ calculated by the Euler-Maruyama scheme with time steps $\Delta t / 2$ and $\Delta t$ respectively. We termed this numerical procedure REM (Richardson extrapolated Euler-Maruyama) scheme, In [42] it is proved that if the drift and diffusion terms of the SDE are sufficiently regular, then the REM scheme has weak convergence of order o( $\left.\Delta t^{2}\right)$, in contrast with the first order (weak convergence) of the Euler-Maruyama scheme, moreover, the extrapolation also improves the stability of the latter. Hence, the REM scheme is computationally more efficient than the Euler-Maruyama scheme.

Let $\tilde{\mathbf{u}}_{\Delta t / 2}^{n}\left(\mathbf{X}^{\mathrm{n}}\right)$ and $\tilde{\mathbf{u}}_{\Delta t}^{n}\left(\mathbf{X}^{n}\right)$ be the solutions at time $t_{n}$ of the SDE Eq. (10) calculated by the Euler-Maruyama scheme with time steps $\Delta t / 2$ and $\Delta t$ respectively, then we take as solution of the SDE at the point $\left(t_{n}, \mathbf{X}^{n}\right)$ the extrapolate

$\mathbf{u}^{\mathrm{n}}\left(\mathbf{X}^{\mathrm{n}}\right)=2 \tilde{\mathbf{u}}_{\Delta t / 2}^{n}\left(\mathbf{X}^{\mathrm{n}}\right)-\tilde{\mathbf{u}}_{\Delta t}^{n}\left(\mathbf{X}^{\mathrm{n}}\right)$.

We must note that in the SDE the tensor terms \{uu $\}$ and $\boldsymbol{\kappa}_{+}$which are to be evaluated at the trajectories $\mathbf{X}(t)$ of the center of mass of the rodlike particles, are approximated in the finite element space $S_{h}^{(2)}$, following the methods proposed in [41]. Specifically, recalling that $\mathbf{K}=(\nabla \mathbf{v})^{T}$, we shall calculate the finite element approximation 
$\boldsymbol{\kappa}_{h}^{n}$ to $\boldsymbol{\kappa}$ at time instant $t_{n}$ as the $L^{2}$-projection of $\left(\nabla \mathbf{v}_{h}^{n}\right)^{r}$ onto the space $S_{h}^{(2)}$; here $\mathbf{v}_{h}^{\text {n }}$ denotes the finite element approximation to $\mathbf{v}$ at time instant $t_{n}$. Thus, letting

$\boldsymbol{\kappa}_{h}^{n}(\mathbf{x})=\sum_{k=1}^{M P}\left(K_{i_{1} i_{2}}^{n}\right)_{k} \Psi_{k}(\mathbf{x}), 1 \leq i_{1}, i_{2} \leq 2$,

where ( $\left.K_{i_{1} i_{2}}^{n}\right)_{k}$ are the values of $\mathbf{\kappa}_{h}^{n}$ at time instant $t_{n}$ at the vertices of the triangles of the mesh, then for each $k$

$\int_{D} \kappa_{h}^{n} \Psi_{k} d \mathbf{x}=\int_{D}\left(\nabla v_{h}^{n}\right)^{r} \Psi_{k} d \mathbf{x}$.

From this equation it follows that the components $\left(K_{\left.i_{1} \tau_{2}\right)_{k}}^{n}\right)_{k}$ are calculated by inverting the so-called mass matrix.

Remark 2. We use the $L^{2}$ projection to recover the second order tensor $\boldsymbol{\kappa}_{h}^{n}=\left(\nabla \mathbf{v}_{h}^{R}\right)^{T}$ for the following reasons:

(1) Looking at the SDE, one needs the coefficients of that equation to be at least Lipschitz continuous for the existence and uniqueness of the solution, with the problem that $\boldsymbol{\kappa}_{h}^{n}$ is discontinuous at the boundaries of the elements; an easy and computationally efficient fashion of recovering $\boldsymbol{\kappa}_{h}^{n}$ as a Lipschitz continuous function, particularly in unstructured meshes, is to $L^{2}$-project it onto the finite element spaces $S_{h}^{(2)}$.

(2) The $L^{2}$-projection with lumping of $\left(\nabla \mathbf{v}_{h}^{n}\right)^{T}$ is an averaging recovery technique. In [43] the efficiency and reliability of averaging techniques as estimators of $(\nabla \mathbf{v})$ is discussed.

(3) It is true, like one of the referees pointed out, that the $\mathrm{L}^{2}$ projection may require finer meshes to represent small scale features of the macro-scale velocity field; however, one may argue that in viscoelastic flows at very low Reynolds number, the macroscale velocity is quite smooth over the whole domain but in certain regions where the mesh is more refined from the start of the calculations.

As for the ensemble average tensor $\{\mathbf{u u}\}$, let $\{\mathbf{u}\rangle_{h}^{n} \in S_{h}^{(2)}$ be its finite element approximation at time $t_{n}$, that is

(u) $\rangle_{h}^{n}(\mathbf{x})=\sum_{k=1}^{M P}\left(T_{i_{1} i_{2}}^{n}\right)_{k} \Psi_{k}(\mathbf{x}), 1 \leq i_{1}, i_{2} \leq 2$.

where $\left(T_{i_{1} j_{2}}^{n}\right)_{k}$ are the values of $\langle\mathbf{u u}\rangle$ at time instant $t_{n}$ at the vertices of the triangles of the mesh, which are obtained by the procedure described in [41] to calculate a finite element approximation to the ensemble average $\langle\boldsymbol{X}$; here $\boldsymbol{\chi}$ can be a tensor of order $r \geq 0$, considering that a scalar function is a tensor of order 0. Let $\chi^{n}\left(\mathbf{X}_{j}^{n}\right)$ denote the value of $\boldsymbol{\chi}$ at the point $\mathbf{X}_{j}^{\mathrm{h}}$ of the trajectory described by the center of mass of the $i$ rodlike particle at time instant $t_{n}$; we approximate the value of the ensemble average of $\chi^{n}\left(\mathbf{X}_{i}^{n}\right)$ at the vertices $\mathbf{x}_{k}$ of the mesh triangles by applying the following scheme:

(1) We divide each triangle of the mesh into three subelements of equal area by joining the barycenter with the vertices. Then, for each vertex $\mathbf{x}_{k}$ we consider the region $A_{k}$ formed by the union of the subelements $A_{k l}$ that share the vertex $\mathbf{x}_{k}$ (see Fig. 2).

(2) In each subelement $A_{k j}$ we find the number of points $\mathbf{X}_{i}^{n}$ lying on it and calculate the ensemble averaged $\left\langle\boldsymbol{X}^{n}\left(\mathbf{X}_{j}^{n}\right)\right\rangle_{\text {- }}$.

(3) $\left\langle\boldsymbol{X}^{n}\right\rangle$ is approximated at the vertex $\mathbf{x}_{k}$ by the area weighted formula

$\chi_{k}^{n}=\frac{1}{\operatorname{Area}\left(A_{k}\right)} \sum_{i=1} \operatorname{Area}\left(A_{k l}\right)\left\langle\chi^{n}\left(\mathbf{X}_{j}^{n}\right)\right\rangle_{i} ;$

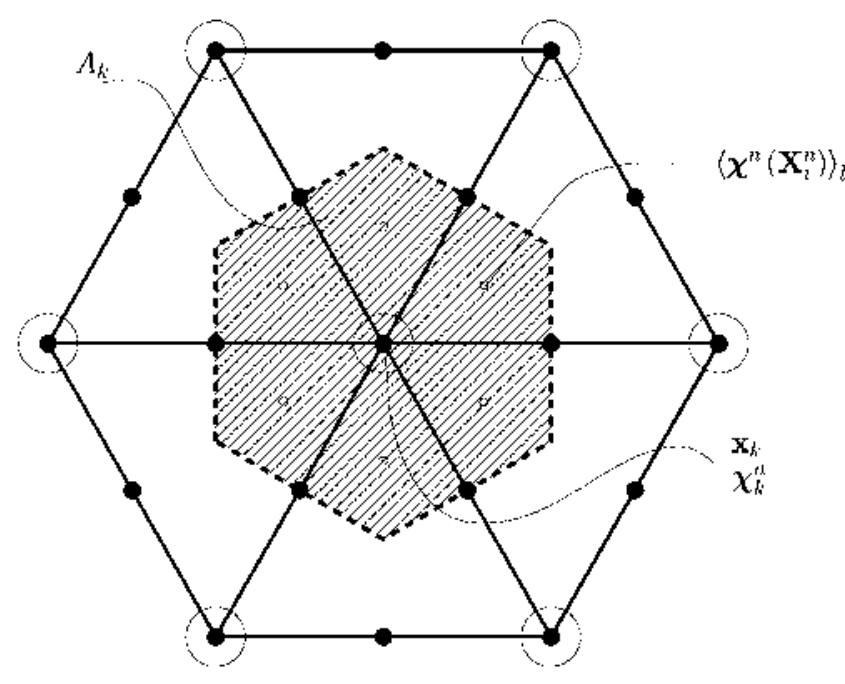

Fig. 2. Scheme for the computation of the $L C$ stress tensor.

the finite element approximation $\langle\boldsymbol{x}\rangle_{h}^{n}$ to $\left\langle\boldsymbol{x}^{n}\right\rangle$ at time $t_{n}$ is then given by

$$
\langle\boldsymbol{X}\rangle_{h}^{n}(\mathbf{x})=\sum_{k-1}^{M P} \boldsymbol{\chi}_{k}^{n} \Psi_{k}(\mathbf{x}) \text {. }
$$

To make simple the presentation of the REM scheme as far as notation is concerned, let us rewrite the SDE equation as

$d \mathbf{u}=\mathbf{b}(\mathbf{u}, \kappa, \mathbf{X}, t) d t+\mathbf{g}(\mathbf{u} . \mathbf{X}, t) \cdot d \mathbf{W}$.

where

$\mathbf{b}(\mathbf{u}, \boldsymbol{\kappa}, \mathbf{X}, t)=\left((\mathbf{I}-\mathbf{u u}) \cdot\left[\mathbf{k} \cdot \mathbf{u} \frac{U}{2 P e}\langle\mathbf{u} \mathbf{u}\rangle \cdot \mathbf{u}\right]-\frac{\mathbf{u}}{3 P e}\right)(\mathbf{X}(t), t)$,

and

$\mathbf{g}(\mathbf{u}, \mathbf{X}, t)=\sqrt{\frac{1}{3 P e}}(\boldsymbol{I}-\mathbf{u u})(\mathbf{X}(t), t)$.

Also, we use the notation $\mathbf{X}^{n}, \mathbf{\kappa}^{n}$, and $\mathbf{u}^{n}\left(\mathbf{X}^{n}\right)$ to denote $\mathbf{X}\left(t_{n}\right)$, $\boldsymbol{\kappa}\left(\mathbf{X}, t_{n}\right)$ and $\mathbf{u}\left(\mathbf{X}\left(t_{n}\right), t_{n}\right)$, respectively. The REM scheme to calculate $\mathbf{X}^{n}$ and $\mathbf{u}^{n}\left(\mathbf{X}^{n}\right)$ for each rodlike particle $i, 1 \leq i \leq N_{p}$, is presented in Algorithm 1, in which $\|\cdot\|$ denotes the modulus of a vector; $\Delta \mathbf{W}_{1}=\mathbf{W}_{t_{\mathrm{n}} 1 / 2}-\mathbf{W}_{t_{n-1}}$ and $\Delta \boldsymbol{W}_{2}=\mathbf{W}_{t_{n}}-\mathbf{W}_{t_{n}{ }_{1 / 2}}$ are the $N(0, \Delta t / 2)$ increments of the three-dimensional Wiener process $\mathbf{w}_{t}$ on $\left[t_{n-1 / 2}, t_{n-1}\right]$ and $\left[t_{n}, t_{n-1 / 2}\right]$, respectively; and $\Delta \boldsymbol{W}_{3}=$ $\Delta \mathbf{W}_{1}+\Delta \mathbf{W}_{2}$ is the $N(0, \Delta t)$ increment of the three-dimensional wiener process $\mathbf{W}_{t}$ on $\left[t_{n}, t_{n} 1\right]$.

\section{Results and discussion}

The method is tested in the same eccentric cylinder geometry as studied in $[22,26]$ (see Fig. 3 ): the outer cylinder remains at rest while the inner cylinder is started impulsively at time $t=0$, rotating with angular frequency $\omega$. Following [22], we select two dimensionless parameters to characterize the geometry shown in Fig. 3: $\mu=\left(R_{2}-R_{1}\right) / R_{1}=7 / 3$ and $\epsilon=e / R_{1}=5 / 3$. We also choose $R_{1}=3$ and $R_{2}=10$ to avoid fractional results; this is equivalent to using $L=R_{1} / 3$ as the characteristic length. Notice also that we study the start-up flow of a LCP solution, since the method presented here is valid for any transient problem.

Three different meshes $M_{1}, M_{2}, M_{3}$ (see Table 1) were considered in the simulations. Convergence was attained with time step sizes $10^{-2} \leq \Delta t \leq 10^{-1}$, eventually choosing $\Delta t \leq 4 \times 10^{-2}$. 


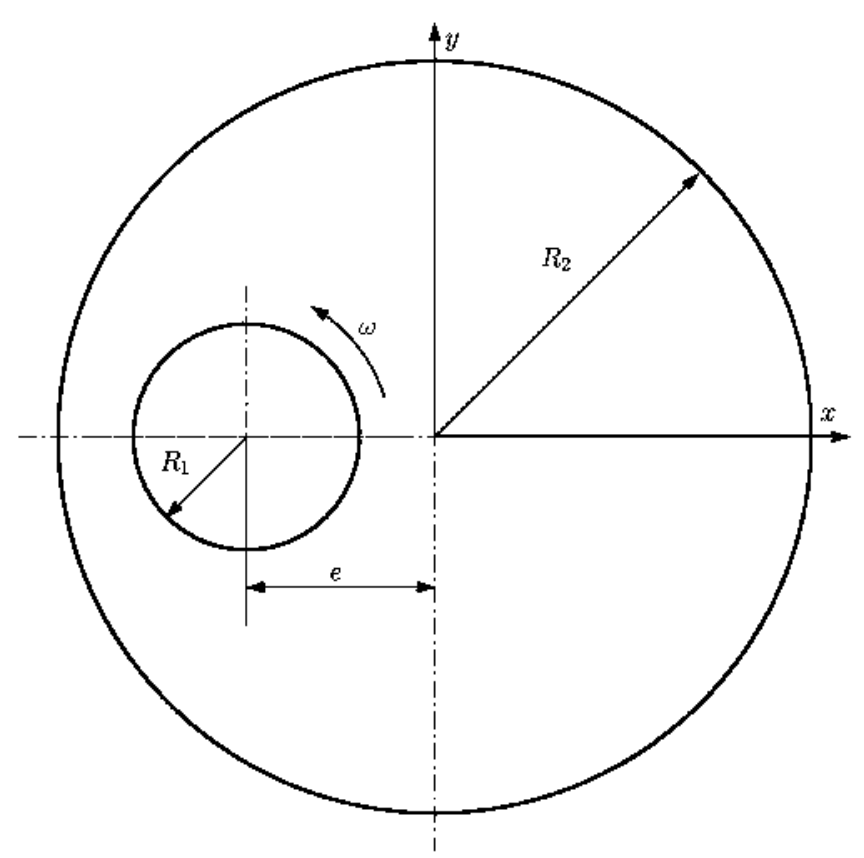

Fig. 3. The eccentric cylinder geometry.

Table 1

Velocity nodes $M V$, pressure nodes $M P$ and number of elements $N E$ for all three considered meshes $M_{1}, M_{2}$ and $M_{3}$.

\begin{tabular}{llll}
\hline Mesh & $M V$ & $M P$ & NE \\
\hline$M_{1}$ & 8984 & 2300 & 4384 \\
$M_{2}$ & 2300 & 602 & 1096 \\
$M_{3}$ & 602 & 164 & 274 \\
\hline
\end{tabular}

Table 2 collects the execution times corresponding to the uncoupled analysis, with final time $T=300$ and step size $\Delta t=4 \times 10^{-2}$, performing 7500 time steps for each run. The most computerintensive calculation, coded in the $C$ programming language, takes roughly two days using a Desktop PC with an Intel Core 2 Duo processor at $2.13 \mathrm{GHz}$ with $2 \mathrm{~GB}$ of RAM, compiled using gcc 4.3 with optimization flag -03 . Memory requirements for the run with the maximum number of particles $N_{p}=5 \times 10^{6}$ reach up to $1.3 \mathrm{~GB}$. It was observed that the refinement of the mesh had an impact not only on the macroscopic variables (velocity, pressure), but on the microscopic magnitudes (scalar order parameter, director vector) as well. Hence, considering the affordable computational times of Table 2, we favor mesh $M_{1}$ and reject meshes $M_{2}$ and $M_{3}$ for the sake of consistency with the numerical scheme, employing a larger number of LCP particles for the density ratio to be maintained. This density number ranges from 300 up to 5000 in the reported simulations with $N_{p}=5 \times 10^{6}$ particles in mesh $M_{1}$, which is about twice as refined as the mesh employed in [22].

Table 2

Execution times (in seconds $\times 10^{4}$ ) for meshes $M_{1} . M_{2}$ and $M_{3}$ for different values of the number of particles $N_{p}=10^{\mathrm{G}}, 2 \times 10^{\mathrm{G}}$ and $5 \times 10^{\mathrm{G}}$.

\begin{tabular}{llll}
\hline Mesh & $N_{p}=10^{6}$ & $N_{p}=2 \times 10^{6}$ & $N_{p}=5 \times 10^{6}$ \\
\hline$M_{1}$ & 3.964032 & 7.090848 & 16.84195 \\
$M_{2}$ & 2.788992 & 5.552064 & 13.76179 \\
$M_{3}$ & 2.632608 & 5.186592 & 11.52144 \\
\hline
\end{tabular}

Algorithm 1. REM scheme for the solution of the SDE

Data Rodlike particle $i$, number of rods $N_{p},\left\{\mathbf{X}_{j}^{n-1}\right\}_{i-1}^{N_{p}}$. $\left\{\mathbf{u}_{j}^{n}{ }^{1}\left(\mathbf{X}_{i}^{n-1}\right)\right\}_{i=1}^{N_{p}}, \mathbf{v}_{h}^{n}{ }^{1}, \mathbf{v}_{h}^{n}{ }^{2}, \boldsymbol{\kappa}_{h}^{n}{ }^{1}$ and $\boldsymbol{\kappa}_{h}^{n}{ }^{1 / 2}$.

Result $\mathbf{X}^{n}$ and $\mathbf{u}^{n}\left(\mathbf{X}^{n}\right)$.

1 Obtain $\mathbf{X}_{i}^{\Pi}$ and $\mathbf{X}_{i}^{\mathrm{n}-1 / 2}$ by the adaptive algorithm of [41].

2 Compute

$$
\begin{aligned}
& \hat{\mathbf{u}}_{\Delta t / 2,1}^{n-1 / 2}\left(\mathbf{X}_{i}^{10-1 / 2}\right)=\mathbf{u}_{i}^{n-1}\left(\mathbf{X}_{i}^{n}{ }^{1}\right) \\
& +\frac{\Delta t}{2} \mathbf{b}\left(\mathbf{u}_{i}, \mathbf{k}_{h}^{l n-1}, \mathbf{X}_{i}^{\mathrm{n}-1}, t_{n-1}\right)+\mathbf{g}\left(\mathbf{u}_{1}, \mathbf{X}_{\mathrm{j}}^{\mathrm{n}-1}, t_{n-1}\right) \cdot \Delta \mathbf{W}_{1},
\end{aligned}
$$

and then impose unit norm condition

$$
\hat{\mathbf{u}}_{\Delta t / 2 . j}^{n-1 / 2}\left(\mathbf{X}_{i}^{n-1 / 2}\right)=\frac{\tilde{\mathbf{u}}_{\Delta t / 2 . j}^{n-1 / 2}\left(\mathbf{X}_{i}^{n-1 / 2}\right)}{\left\|\tilde{\mathbf{u}}_{\Delta t / 2 . j}^{n-1 / 2}\left(\mathbf{X}_{i}^{n-1 / 2}\right)\right\|} .
$$

\section{Compute}

$$
\begin{aligned}
\tilde{\mathbf{u}}_{\Delta t / 2 . j}^{n}\left(\mathbf{X}_{i}^{n}\right) & =\tilde{\mathbf{u}}_{\Delta t / 2 . i}^{n-1 / 2}\left(\mathbf{X}_{i}^{n-1 / 2}\right)+\frac{\Delta t}{2} \mathbf{b}\left(\mathbf{u}_{i}, \mathbf{\kappa}_{h}^{n-1 / 2}, \mathbf{X}_{i}^{n-1 / 2}, t_{n-1 / 2}\right) \\
& +\mathbf{g}\left(\mathbf{u}_{j}, \mathbf{X}_{i}^{n-1 / 2}, t_{n-1 / 2}\right) \cdot \Delta \mathbf{W}_{2},
\end{aligned}
$$

and impose

$$
\hat{\mathbf{u}}_{\Delta t / 2, j}^{n}\left(\mathbf{X}_{i j}^{n}\right)=\frac{\tilde{\mathbf{u}}_{\Delta t / 2, i}^{n}\left(\mathbf{X}_{j}^{n}\right)}{\left\|\tilde{\mathbf{u}}_{\Delta t / 2, i}^{n}\left(\mathbf{X}_{i}^{n}\right)\right\|} .
$$

\section{Compute}

$$
\begin{aligned}
\tilde{\mathbf{u}}_{\Delta t, i}^{n}\left(\mathbf{X}_{i}^{n}\right) & =\mathbf{u}_{i}^{n-1}\left(\mathbf{X}_{i}^{n-1}\right)+\Delta t \mathbf{b}\left(\mathbf{u}_{i}, \mathbf{\kappa}_{h}^{n-1}, \mathbf{X}_{i}^{n-1}, t_{n} 1\right) \\
& +\mathbf{g}\left(\mathbf{u}_{i}, \mathbf{X}_{i}^{n-1}, t_{n-1}\right) \cdot \Delta \mathbf{W}_{3},
\end{aligned}
$$

and impose

$\hat{\mathbf{u}}_{\Delta t, i}^{n}\left(\mathbf{X}_{i}^{n}\right)=\frac{\tilde{\mathbf{u}}_{\Delta t, i}^{n}\left(\mathbf{X}_{i}^{n}\right)}{\left\|\tilde{\mathbf{u}}_{\Delta t, i}^{n}\left(\mathbf{X}_{i}^{n}\right)\right\|}$.

5 Apply extrapolation and impose unit norm:

$$
\hat{\mathbf{u}}_{i}^{n}\left(\mathbf{X}_{i}^{n}\right)=2 \hat{\mathbf{u}}_{\Delta t / 2 . j}^{n}\left(\mathbf{X}_{i}^{n}\right)-\hat{\mathbf{u}}_{\Delta t, i}^{n}\left(\mathbf{X}_{i}^{n}\right) ; \quad \mathbf{u}_{i}^{n}\left(\mathbf{X}_{i}^{n}\right)=\frac{\hat{\mathbf{u}}_{i}^{n}\left(\mathbf{X}_{i}^{n}\right)}{\left\|\hat{\mathbf{u}}_{i}^{n}\left(\mathbf{X}_{i}^{n}\right)\right\|} .
$$

\subsection{Uncoupled analysis}

We first consider the decoupled situation as presented by Grosso et al. [26] in their discussion of different closure approximations for the Hess-Doi model. In that work, the authors perform several simulations in a decoupled fashion, solving the Fokker-Planck equation and then using the kinetic information as an input to their macroscopic module. Since our method can cope with this situation by setting the coupling (or concentration) parameter $c=0$, we have run simulations for $P e=10,20$, and also for $P e=1,5,50$ and 100 , choosing the interaction potential $U=5.392$ as that corresponding to the same equilibrium value of the scalar order parameter $S_{\text {eq }}=0.68(3)$. We follow $[22,26,33]$ and define the scalar orientational order parameter by $S=[(3 \mathbf{A}: \mathbf{A}-1) / 2]^{1 / 2}$, where $\mathbf{A}=\langle\mathbf{u u}\rangle$ is the configuration tensor.

Remark 3. A more appropriate definition for $S$ is given by $S=$ (3/2)dd : S, which allows for negative values of the scalar order parameter occurring in planar biaxial ordered states, e.g. near defects. In Fig. 16, we show that both definitions of $S$ agree very well 


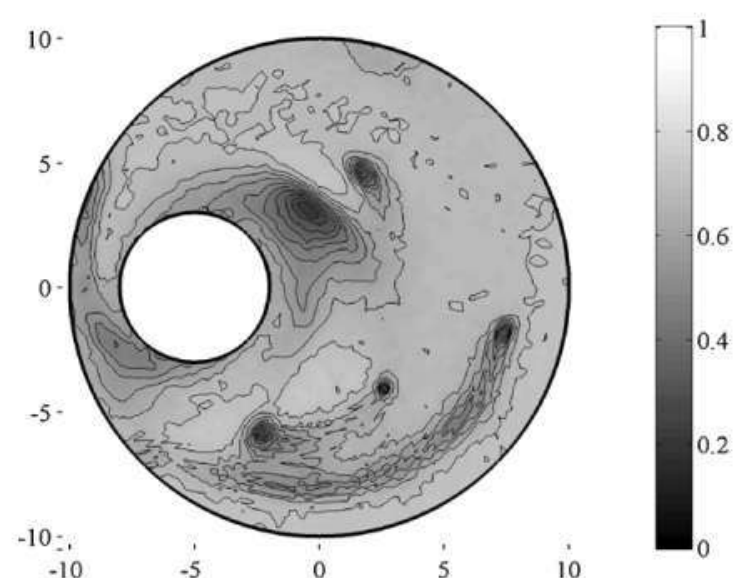

Fig. 4. Field of the scalar order parameter $S$ for $c=0, P e=1, U=5.392$.

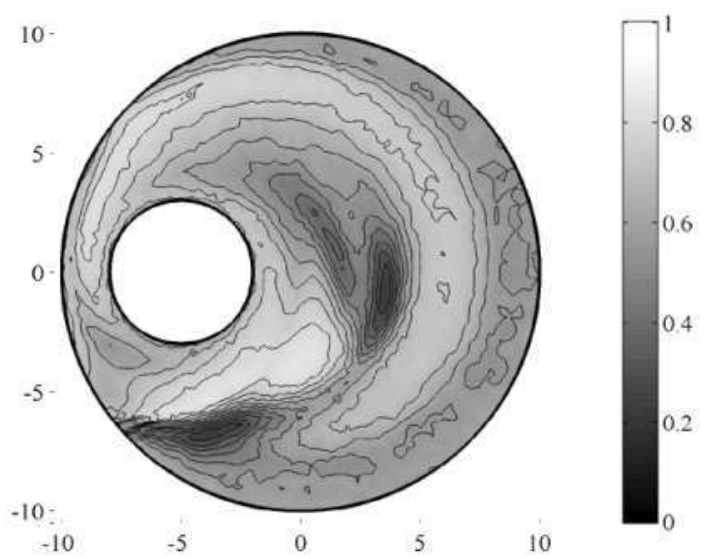

Fig. 5. Field of the scalar order parameter $S$ for $c=0, P e=10, U=5.392$.

at points $A, B$ and $C$ for the uncoupled, local analysis. In the following, we keep the original definition of $S$ for comparison purposes with [22] and [26].

The initial distribution of configurations is sampled from a disordered state, the purpose being to compare the Brownian approach with the closure approximations of that paper.

The field of the scalar order parameter $S$ for certain values of $\mathrm{Pe}$ at steady state is shown in Figs. 4-6. Note: 'FPE' data refers to that obtained by [26] for the Fokker Planck Equation by means of the spherical harmonics technique. It may be observed a close resem-

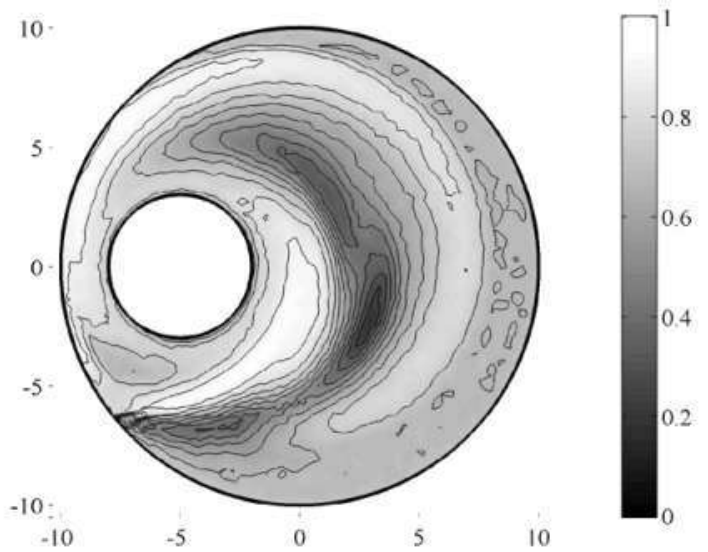

Fig. 6. Field of the scalar order parameter $S$ for $c=0, U=5.392$.

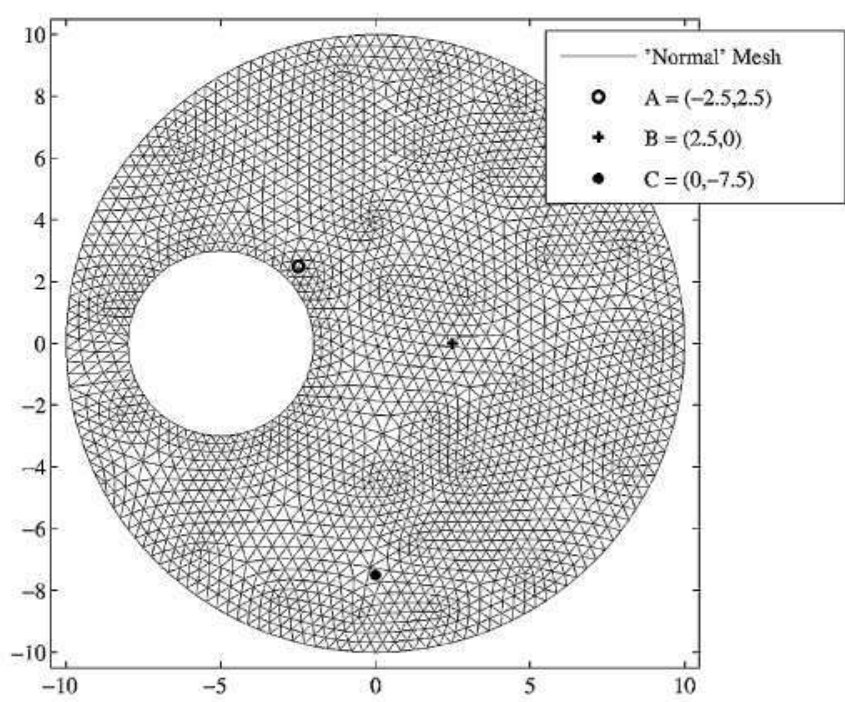

Fig. 7. Points $A \equiv(-2.5,2.5), B \equiv(2.5,0), C \equiv(0,-7.5)$ for the measurement of $S$ and $\alpha$ (with respect to the $x$ axis), for $c=0, U=5.392$, in mesh $M_{1}, \Delta t=4 \times 10^{-2}$. $N_{p}=5 \times 10^{6}$ particles.

blance with the field provided by the reference ('FPE') solution for $P e=10$, whereas the quadratic closure approximation from [26] offers larger and smoother values. The scalar order parameter field shows a change in character from $P e=1$ to $P e=10$, then remaining qualitatively the same up to $P e=100$. This transition may be more readily noticed by a local analysis of the field, as suggested by Grosso et al.

The local analysis is performed by selecting three different points of the domain $A, B, C$ (see Fig. 7). Results for the scalar order parameter $S$ as well as the orientation $\alpha$ of the director field $\mathbf{d}$, measured with the $x$-axis, are provided in Figs. 8-15 for the selected Peclet numbers. In this work, as usual, the director field d is represented by means of ellipses in which the width and length are proportional to the magnitude of the two eigenvalues associated to the eigenvectors of the configuration tensor A placed in the flow plane, and the orientation is provided by the eigenvector associated to the eigenvalue of the largest magnitude.

In points $A$ and $C$, our method predicts stationary values almost identical to the ones provided by [26] for $P e=10,20$, both for the scalar order parameter $S$ and the director orientation $\alpha$. The aforementioned transition is now observed at point $B$ for $P e=1$ and

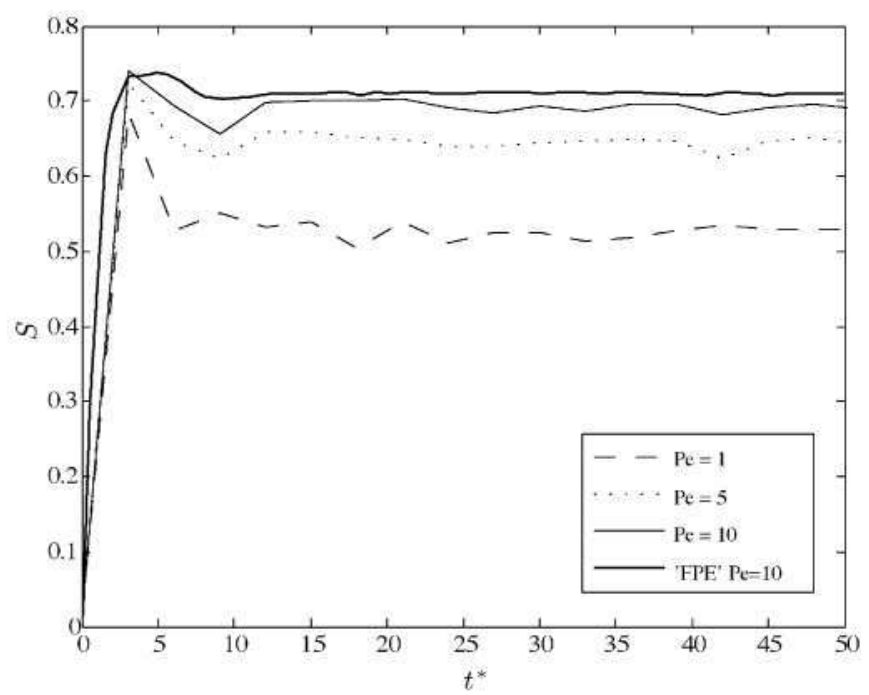

Fig. 8. Scalar order parameter $S$ at point $A . P e=1,5,10$, 'FPE'. 


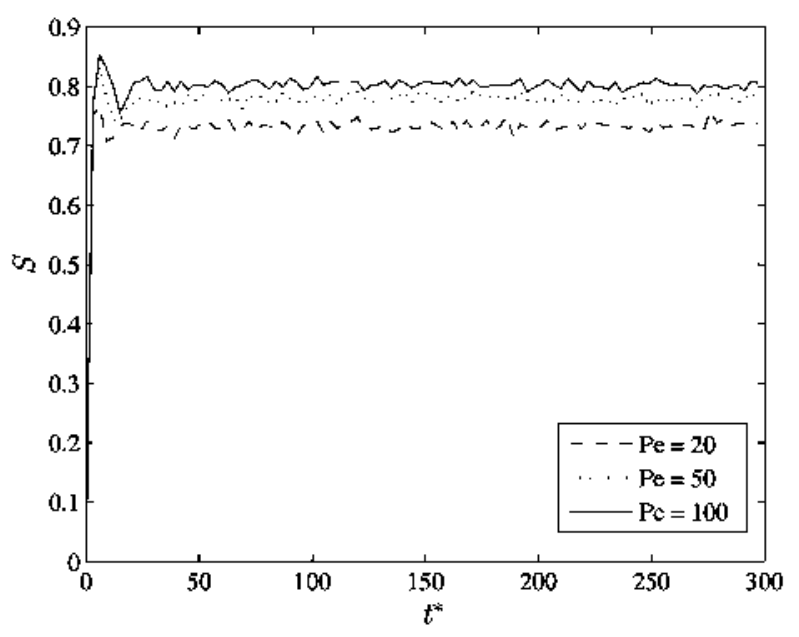

Fig. 9. Scalar order parameter $S$ at point $A . P e=20,50.100$.

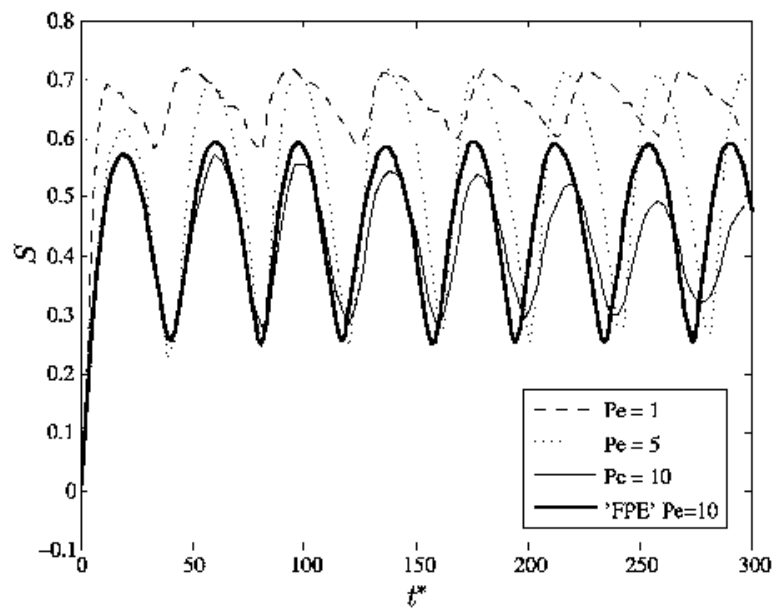

Fig. 10. Scalar order parameter $S$ at point B. Pe =1,5. 10, 'FPE'.

larger: from a tumbling-like solution, similar to that predicted by the Bingham closure for $P e=10$ in [26], to a wagging behavior predicted by our model for $P e=5$, to a damped oscillation for $P e \geq 10$. Damped oscillations are experimentally observed for LCP solutions and are also reported by Grosso et al. for $P e=20$; in our study, we observe this transition from wagging to damped oscillations at smaller values of $P e$.

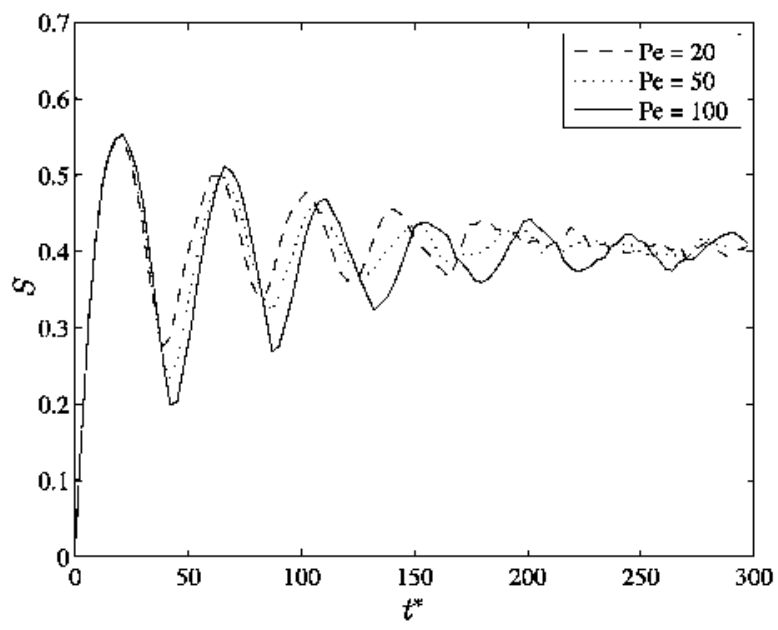

Fig- 11. Scalar order parameter $S$ at point $B . P e=20,50,100$.

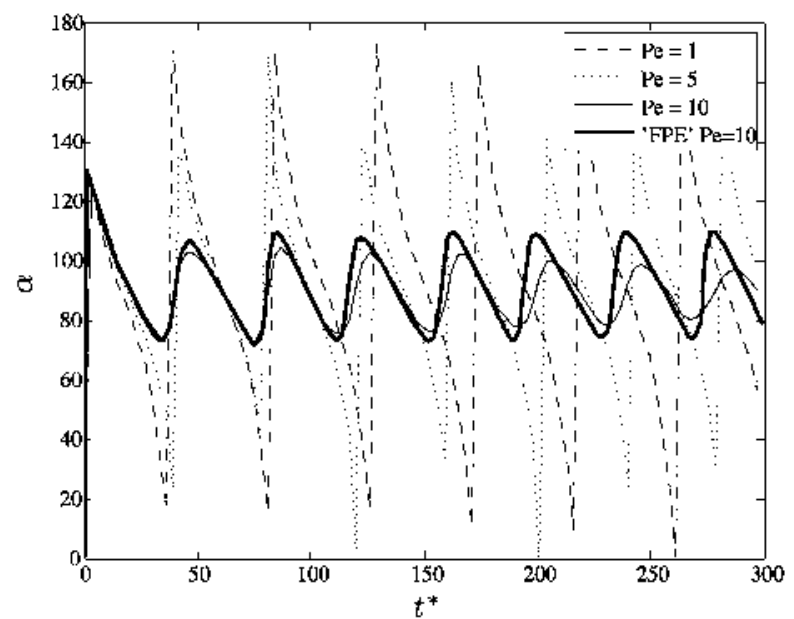

Fig. 12. Director orientation $\alpha$ at point $B . P e=1,5,10$, 'FPE'.

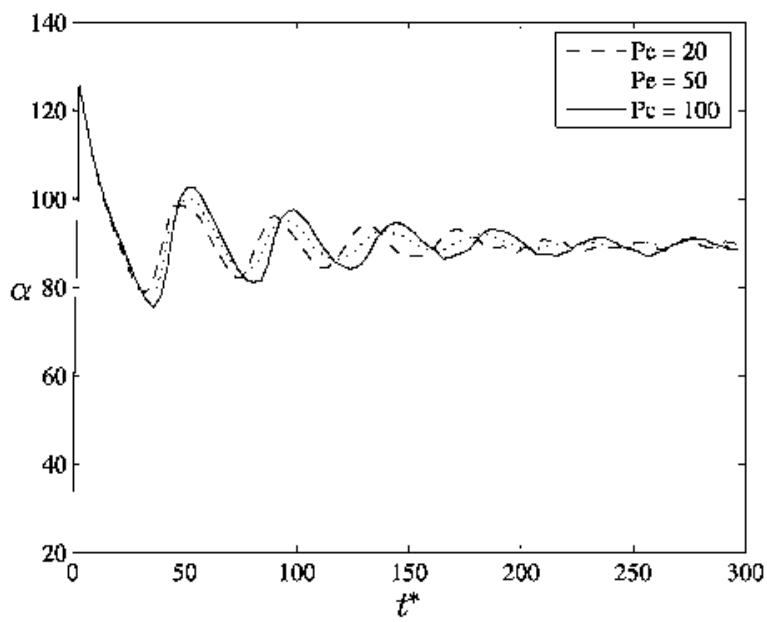

Fig- 13. Director orientation $\alpha$ at point $\mathrm{B} . \mathrm{Pe}=20,50,100$.

A possible explanation could be as follows: the macroscopic part of the problem can play such a role in the computation of the stochastic magnitudes that slight variations in the velocity gradient at certain points of the flow may change the character of the microscopic magnitudes. This hypothesis is at least plausible since, for simple shear, non-equilibrium phase transitions between different

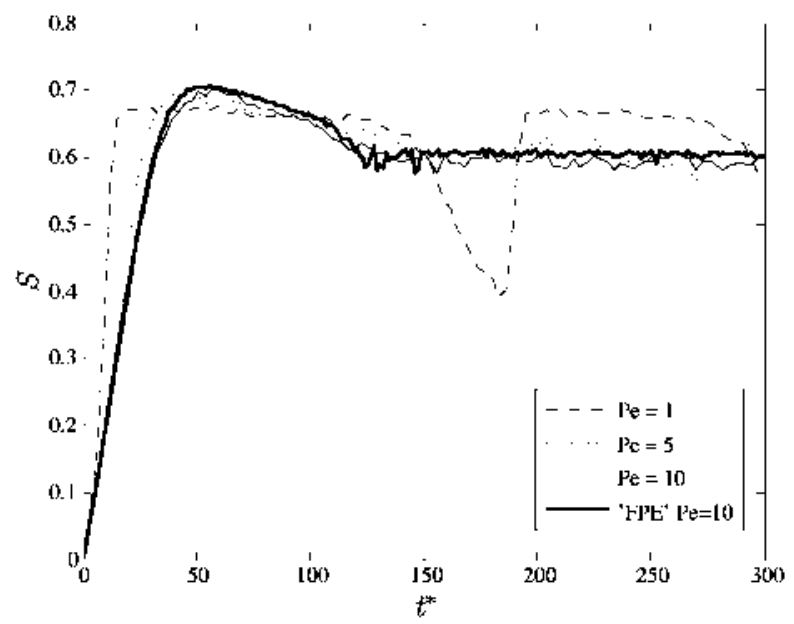

Fig. 14. Scalar order parameter $S$ at point $C . P e=1,5,10$, 'FPE'. 


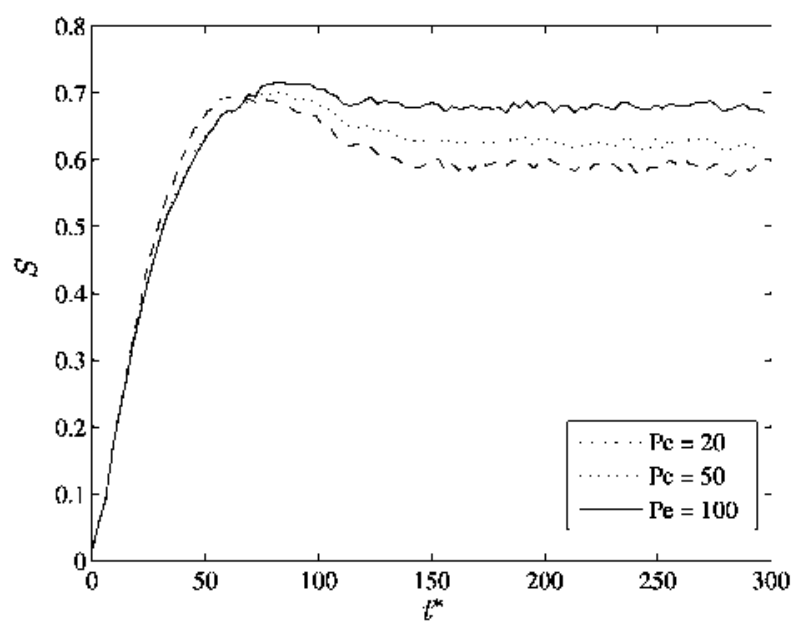

Fig. 15. Scalar order parameter $S$ at point C. $P e=20.50 .100$.

dynamical states are known to occur upon slight variations of shear rate [14]. To further verify this hypothesis, we conducted purely Brownian simulations with fixed values of the velocity gradient $\boldsymbol{\kappa}+\Delta \boldsymbol{\kappa}$ slightly deviating from the accurately computed velocity gradient $\mathbf{\kappa}$ (employing meshes with a number of elements in the problematic region far exceeding that of mesh $M_{1}$ ); the results, as shown in Fig. 17, support the previous explanation. Furthermore, a convergence check was carried out by means of purely stochastic simulations, taking as an input the velocity gradient at point $B$, varying the ensemble (here using no macroscopic mesh whatsoever) and the time step size for both non-extrapolated and Richardson extrapolated Euler-Maruyama schemes; the damping was predicted in all cases for $P e=10$ (see Figs. 18 and 19).

\subsection{Coupled analysis}

Next, we activate the coupling or backflow effect between the LCP and the macroscopic flow, by setting the concentration number $c$ to a non-zero value, taking as a reference the work by Feng and Leal [22] for comparison purposes. The Reynolds number $R e=1.11 \times$ $10^{4}$ as well as the Peclet $P e=20$, the empirical value $\beta=10^{3}$, the prefactor $\left(n L^{3}\right)^{2}=2 \times 10^{5}$, and the intensity of the dimensionless interaction potential $U=4$, were fixed to the same values used in [22], with such a small Reynolds number being chosen so as to avoid

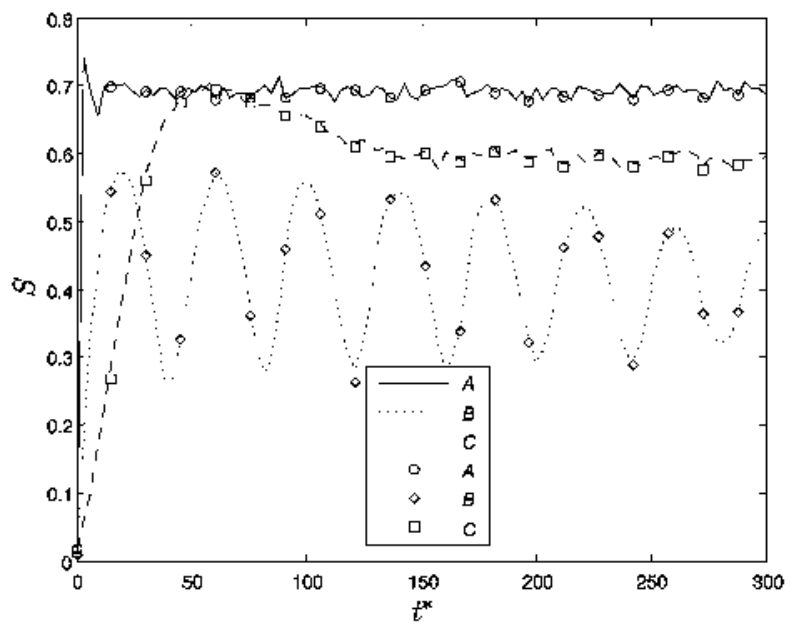

Fig. 16. Comparison between different definitions of $S$ at points $A . B$ and $C . S=\frac{3}{2}$ dd : $\mathbf{S}$ (maskers) and $S=(3(\mathbf{A}: \mathbf{A}-1) / 2)^{1 / 2}$ (solid line).

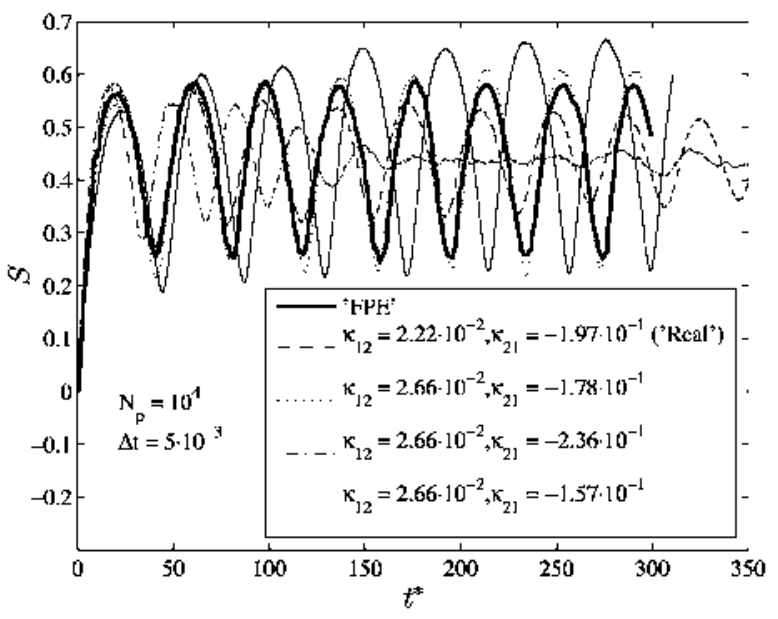

Fig. 17. Deviation of the scalar order parameter at point $B$ from baseline values after a slight variation of the transposed velocity gradient $k$ for an ensemble of $N_{p}=10^{4}$ particles with $\Delta t=5 \times 10^{-3}$.

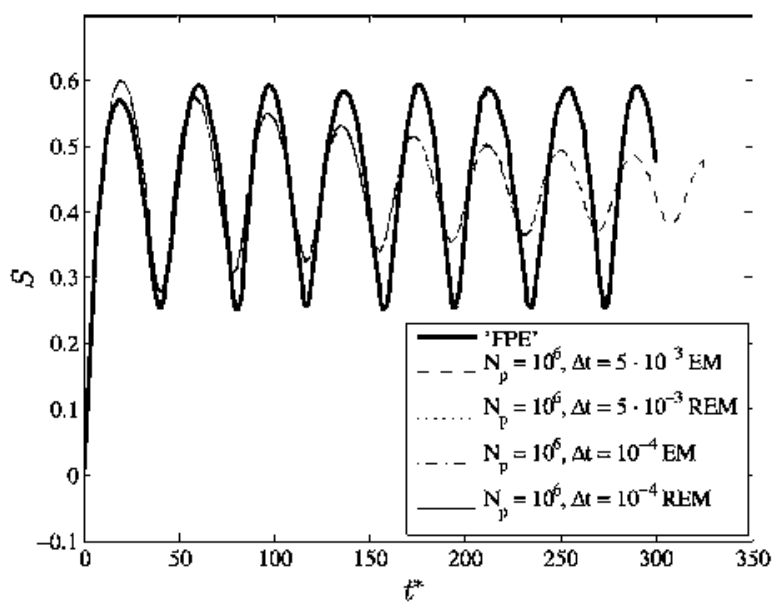

Fig. 18. Convergence of the scalar order parameter at point $B$ as a function of the time step, for Euler-Maruyama and Richardson extrapolated Euler-Maruyama schemes. $N_{p}=10^{6}$ particles.

any transient state. Initially, the rodlike particles were aligned with the horizontal $x$-axis. We choose $c=10,100,150$ and 200, keeping the influence of the LCP on the flow limited to a certain degree; the evolution of the $S$ field with an increase in the concentration

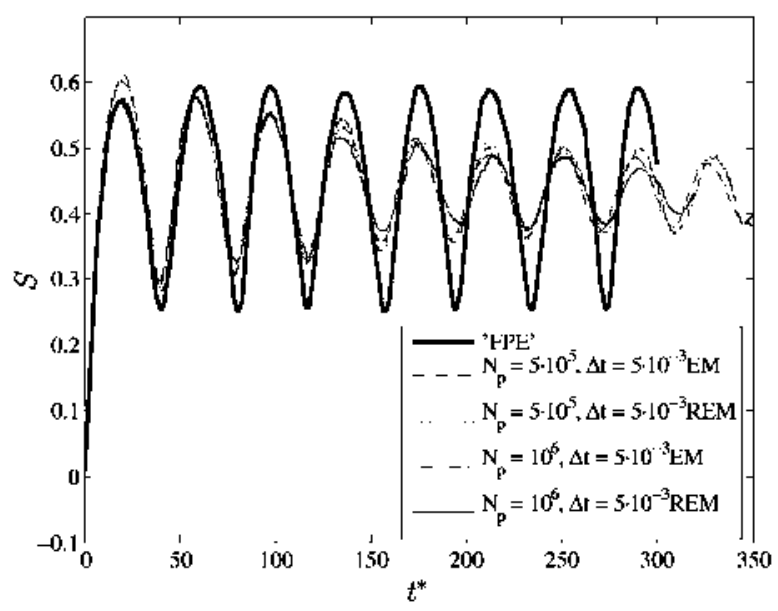

Fig. 19. Convergence of the scalar order parameter at point $B$ with ensemble size for Euler-Maruyama and Richardson extrapolated Euler-Maruyama schemes. Time step $\Delta t=5 \times 10^{-3}$. 


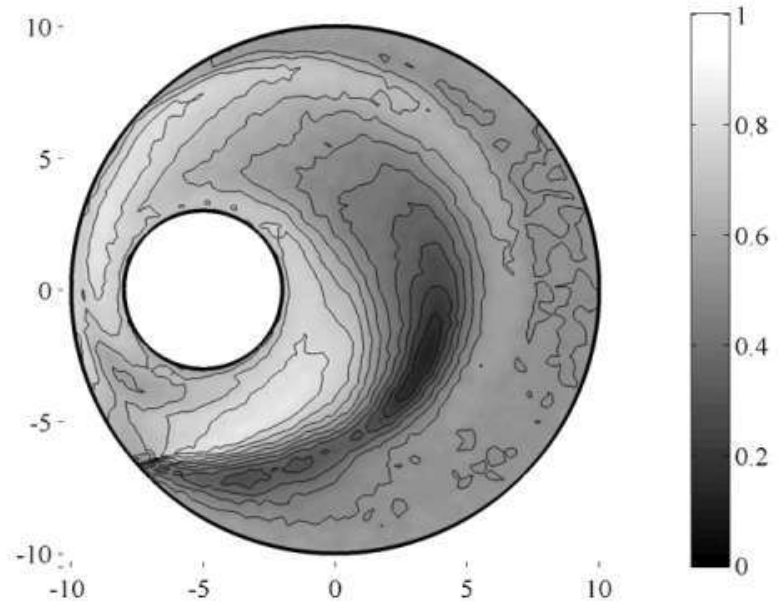

Fig. 20. Field of the scalar order parameter $S$ for $c=10, P e=20, U=4, \Delta t=4 \times$ $10^{-2}$.

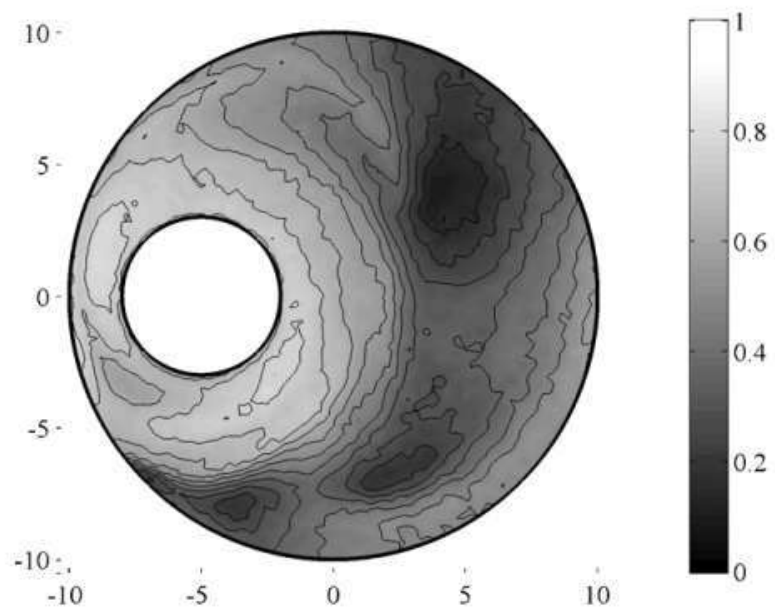

Fig. 21. Field of the scalar order parameter $S$ for $c=100, P e=20, U=4, \Delta t=4 \times$ $10^{-2}$.

number $c$ is shown in Figs. 20-23: in the regions close to the inner cylinder, $S$ remains rather unchanged for all $c$ values, whereas an abrupt reduction is observed at zones $x \geq 0$.

The values predicted by our model for the scalar order parameter are somewhat lower than those reported in [22] using the quadratic closure; however, this closure is known to provide smoother and

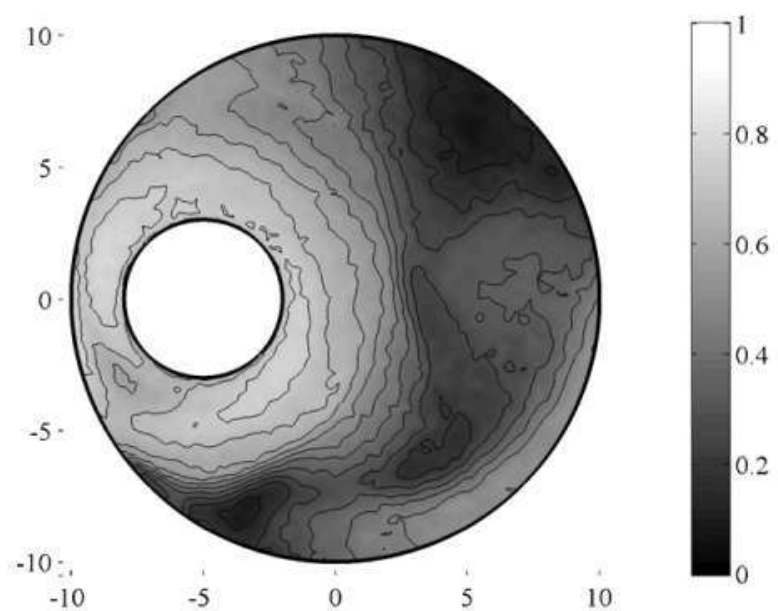

Fig. 22. Field of the scalar order parameter $S$ for $c=150, P e=20, U=4, \Delta t=2 \times$ $10^{-2}$.

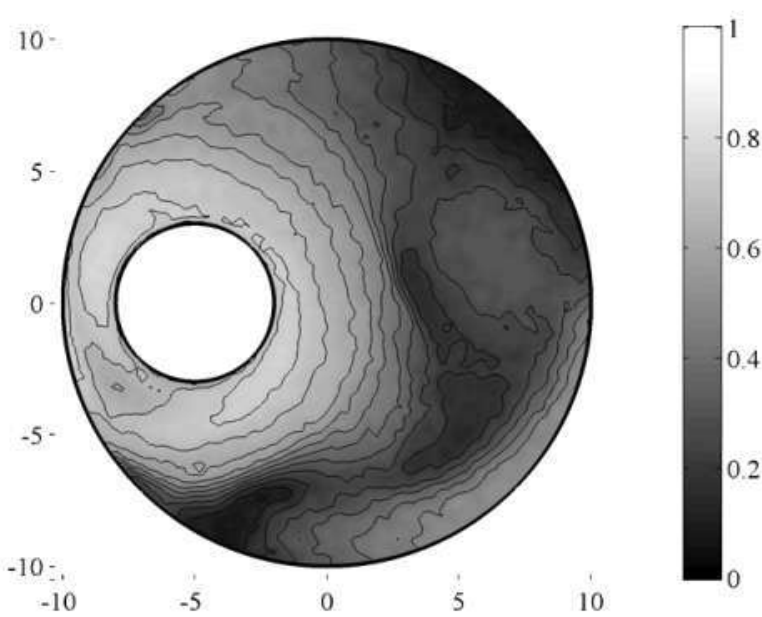

Fig. 23. Field of the scalar order parameter $S$ for $c=200, P e=20, U=4, \Delta t=2 \times$ $10^{-2}$

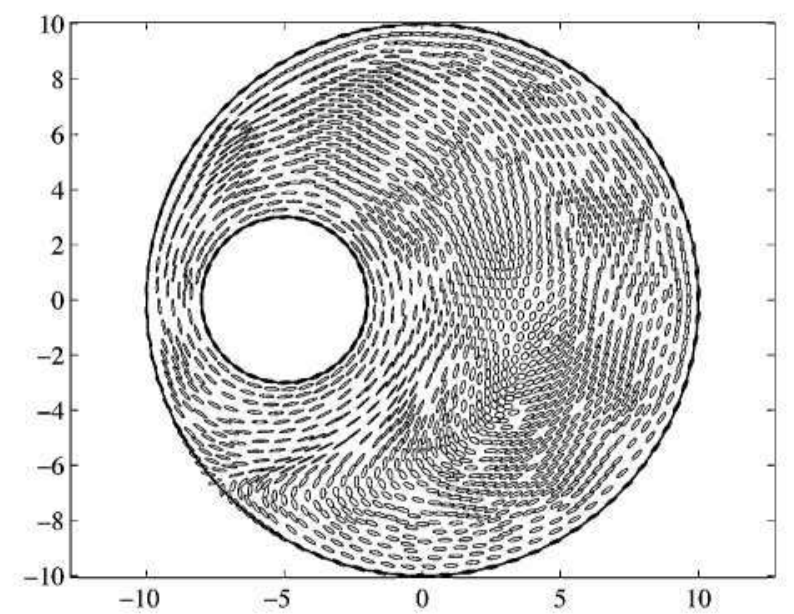

Fig. 24. Director vector $\mathbf{d}$ for $\Delta t=4 \times 10^{-2}, c=10$ with mesh $M_{1}, P e=20, U=4$, $N_{p}=5 \times 10^{6}$.

overestimated values with respect to those predicted by the more accurate Bingham closure (see, e.g. [26] for a detailed discussion). The director field $\mathbf{d}$ for the lowest and highest values of $c$ is also represented in Figs. 24 and 25; the disclination lines observed by [22] and [25] are apparent for the case of $c=10$, whereas for $c=$ 200 none could be distinctly observed.

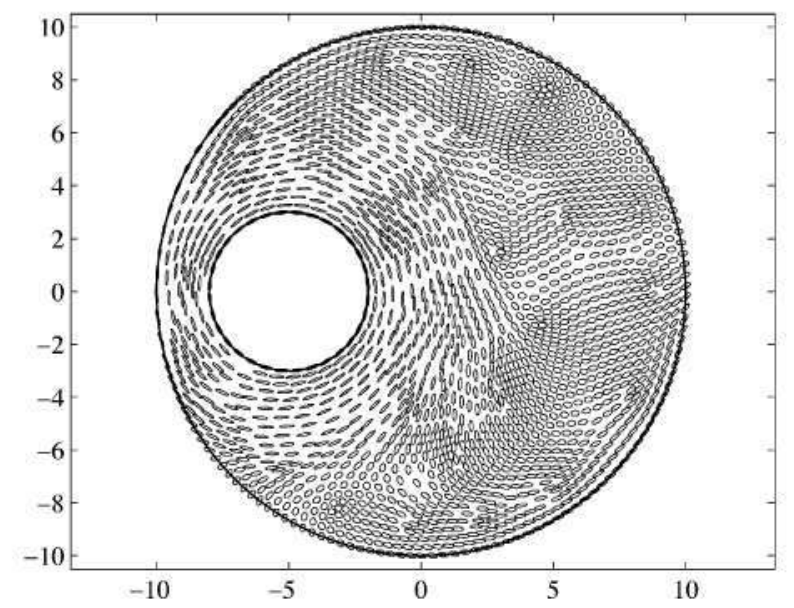

Fig. 25. Director vector $\mathbf{d}$ for $\Delta t=2 \times 10^{-2}, c=200$ with mesh $M_{1}, P e=20, U=4$, $N_{\mathrm{p}}=5 \times 10^{6}$ 


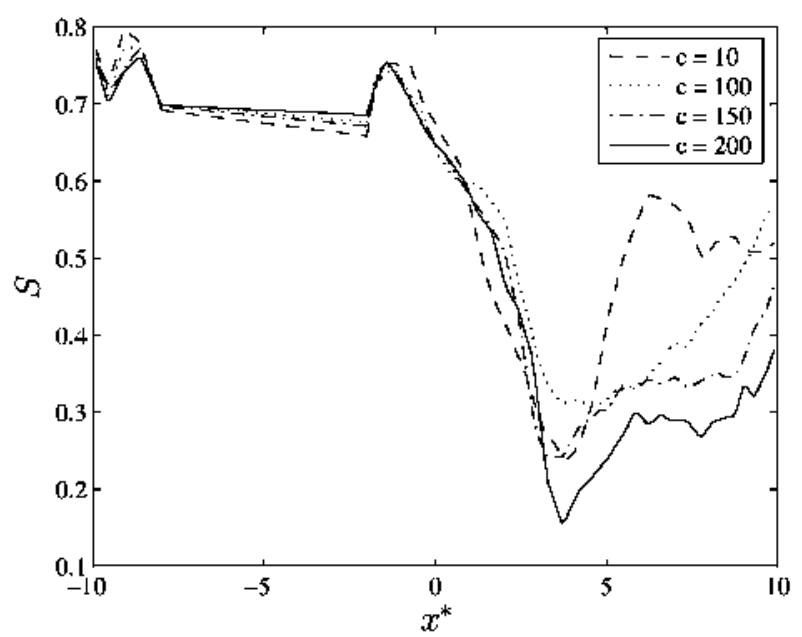

Fig. 26. Order parameter $S$ along the $x$ axis for $c=10,100,150$ and $200 ; U=4$; $P e=20 ;$ mesh $M_{1}: \Delta t=4 \times 10^{-2} ; N_{p}=5 \times 10^{6}$.

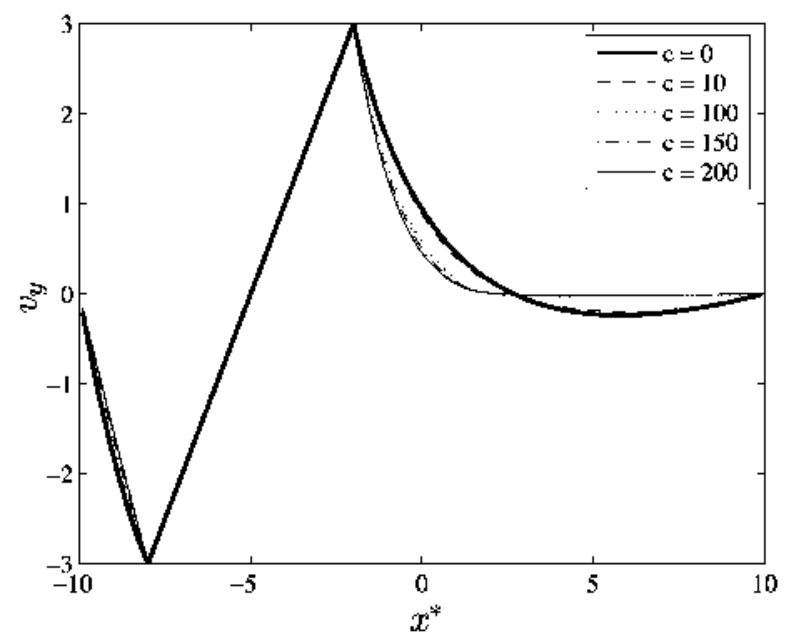

Fig. 27. Vertical component of the velocity, $v_{y}$ along the $x$ axis for $c=10,100,150$ and 200; $U=4 ; \mathrm{Pe}=20$; mesh $M_{1} ; \Delta t=4 \times 10^{2} ; N_{p}=5 \times 10^{\mathrm{t}}$.

An investigation of $S$ as well as the vertical component of the velocity $v_{y}$ and the magnitude of the strain-rate tensor $\dot{\gamma}=$ (D : D $)^{1 / 2}$, where $\mathbf{D}=\left(\nabla \mathbf{v}+(\nabla \mathbf{v})^{T}\right) / 2$, was performed along the horizontal $x$ axis of the eccentric cylinder (see Figs. 26-28, respec-

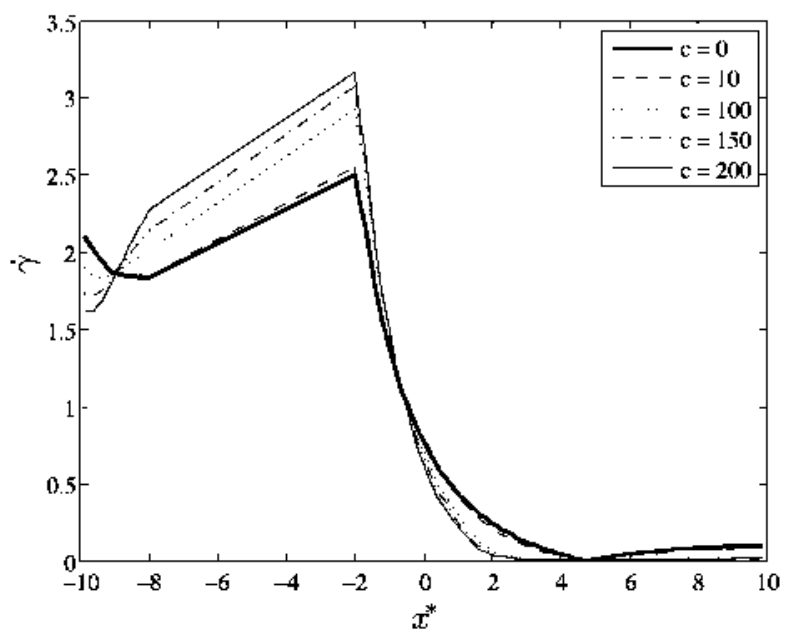

Fig. 28. Magnitude $\dot{\gamma}$ of the rate-of-strain tensor along the $x$ axis for $c=10.100$. 150 and $200 ; U=4 ; P e=20 ;$ mesh $M_{1} ; \Delta t=4 \times 10^{2} ; N_{p}=5 \times 10^{6}$. tively). Now, the behavior of $S$ is more easily recognizable according to the pattern expressed above: attenuation as $c$ grows up, a feature also recorded by Feng and Leal in their study. As for the vertical component of the velocity, the influence of the polymer is apparent, producing an "abatement" of the flow in the proximity of the inner cylinder, growing in intensity as $c$ increases, much in accordance with the predictions of [22]. The results for the magnitude of the strain-rate $\dot{\gamma}$ present a reciprocal behavior, increasing the rate of strain with the coupling between polymer and flow.

\section{Conclusions}

A comparative study on complex flows of liquid-crystalline solutions using a semi-Lagrangian micro-macro method has been presented. The method has been tested with a reference problem, namely, the eccentric rotating cylinder, the analysis being carried out in two parts.

Firstly, the stochastic part of the model is highlighted by assessing the situation from a decoupled point of view, as suggested by [26]. Investigations of the Peclet number from $P e=1$ and up to values $P e=100$ are reported, showing a dramatic change in the behavior of the scalar order parameter in the non-stationary zones. A local analysis distinctly records that transition: from wagging to tumbling to damped oscillations at point $B$. We find the transition from wagging to damped oscillations at somewhat lower Peclet numbers compared to [26] and offer plausible arguments for this difference. Purely stochastic simulations confirm convergence and damping of the oscillations for $P e \geq 10$ for the examined situations.

Secondly, the effect of the LCP on the flow is investigated, and microscopic and macroscopic variables are compared to those provided by [22] for $c=10.100$, while also providing results for $c=150$ and $c=200$. An abatement of the flow and reduction of the scalar order parameter field are observed when increasing the coupling. Moreover, disclinations for $c=10$ are also registered at two located regions of the flow, gradually disappearing as $c$ exceeds $c=100$.

The scheme is a general, bi-dimensional, second order accurate in time and space semi-Lagrangian micro-macro method, which to the best of our knowledge has been employed for the first time here to predict rheological behavior of liquid crystalline solutions both in transient and stationary problems. The results provided for the eccentric rotating cylinder geometry show their ability to cope with complex flows and presents itself as an alternative to LCP flow simulations with closure approximations.

\section{Acknowledgments}

The authors would like to thank the reviewers for their suggestions and comments which have helped to improve the original manuscript.

Financial support by the EC through contracts G5RD-CT-200200720 and NMP3-CT-2005-016375, partial support by CICYT grant MAT2006-04029, and support from the Barcelona Supercomputing Center and the high performance computational cluster Magerit at CesviMa UPM is acknowledged.

\section{References}

[1] F.M. Leslie, Theory of flow phenomena in liquid crystals, Adv. Liq. Cryst. 4(1979) $1-81$

[2] R.G. Larson, Roll-cell instabilities in shearing flows of nematic polymers, ] Rheol. 37 (2) (1993) 175-197.

[3] R.G. Larson, M. Doi, Mesoscopic domain theory for textured liquid crystalline polymers, J. Rheol. 35 (4) (1991) 539-563.

[4] B.]. Edwards, A.N. Beris, M. Grmela, Generalized constitutive equation for polymeric liquid crystals. 1. Model formulation using the Hamiltonian (Poisson bracket) formulation. ]. Non-Newtonian Fluid Mech. 35 (1990) 51-72. 
[5] S. Hess, Fokker-Planck equation approach to flow-alignment in liquid crystals. Z. Naturforsch 31a (1976) 1034-1037.

[6] M. Doi, S. Edwards, The Theory of Polymer Dynamics, Clarendon Press, oxford, 1986.

[7] R.G. Larson, H.C. Öttinger, Effect of molecular elasticity on out-of-plane orientations in shearing flows of liquid crystalline polymers, Macromolecules 24 (1991) 6270-6282.

[8] T. Maruyama, G.G. Fuller, M. Grosso, P.L. Maffetone, The dynamics of two dimensional polymer nematics, J. Non-Newtonian Fluid Mech. 76 (1998) $233-247$.

[9] M.G. Fortest, Q. Wang. H. Zhou, Exact banded patterns trom a Doi-MartucciGreco model of nematic liquid crystal polymers, Phys. Rev. E 61 (6) (2000) $6655-6662$.

[10] G. Sgalari, L.G. Leal, J.t. Feng. The shear flow beliavior of LCPS based on a generalized Doi model with distortional elasticity, J. Non-Newtonian Flu id Mech. 102 (2002) 361-382.

[11] M.C. Forest, R. Zhou, Q. Wang, Full-tensor alignment criteria for sheared nematic polymers, J. Rheol. 47 (1) (2003) 105-127.

[12] M. Grosso, S. Crescitelli, E. Somma, J. Vermant, P. Moldenaers, P.L. Matfetone. Prediction and observation of sustained oscillations in sheared liquid crystalline polymer, Pliys. Rev. Lett. 90 (2003) 098304 .

[13] M.G. Forest, Q Wang, R. Zhou, The weak shear phase diagram for nematic polymers, Rheol. Acta 43 (2004) 17-37.

[14] M.G. Forest, $Q$, Wang. R. Zhou, The flow-phase diagram of Doi-Hess theory tor sheared nemat ic polymers. Il. Finite shear rates, Rheol. Acta 44(1)(2004)80-93.

[15] R.H. Zhou, M.G. Forest, Q, Wang. Kinetic structure simulations of nematic polymers in plane Couette cells. I. The algorithm and benchmarks, Multiscale Model. Simul. 3(4) (2005) $853-870$.

[16] P. Ilg. S. Hess, Two-alignment tensor theoty tor the dynamics of side chain liquid-crystalline polymers in planar shear flow, J. Non-Newtonian Fluid Mech. $134(1-3)$ (2006) 2-7.

[17] R,H. Zliou, M,G. Forest, Q, Wang, Kinetic structure simulations of nematic polymers in plane Couette cells. II: in-plane structure transitions, Multiscale Model. Simul. 4 (4) (2005) 1280-1304.

[18] S. Grandner, S. Heidenreich, P. Ilg, S.H.L. Klapp. S. Hess, Dynamic electric polarization of nematic liquid crystals subjected to a sheat flow, Pliys. Rev. E 75 (2007) $040701 \mathrm{R}$.

[19] C. Denniston, E. Orlandini, J.M. Yeomans, Simulations of liquid crystals in Poiseuille flow, Comput. Theor. Polymer Sci. 11 (2001) 389-395.

[20] Z. Cui, M.G. Forest, Q Wang, R.H. Zhou, on weak plane Couette and Poiseuille flows of tigid cod and platelet ensembles, SIAM J. Appl. Matli. 66 (4) (2006) $1227-1260$.

[21] J. Feng, L.C. Leal, Pressure-driven channel flows of a model liquid-crystalline polymer, Plyys. Fluid. 11 (10) (1999) 2821-2835.

[22] J. Feng, L.G. Leal, Simulating complex flows of liquid-crystalline polymers using the Doi theory. J. Rheol. 41 (1997) 1317-1335.

[23] C.V. Chaubal, L.G. Leal, G.H. Fredrickson, A comparison of closure approximations for the Doi theory of LC.P5, J. Rheol. 39 (1995) 73-103.

[24] M. Kröger; A Ammar, F. Chinesta, Consistent closure schemes for statistical models of anisotropic fluids. J. Non-Newtonian Fluid Mech. 149 (2008) 40-55.
|25| J. Feng, C.V.Cliaubal, L.G. Leal, Closute apptoximat ions tor the Doi theory: which to use in simulating complex flows of liquid-crystalline polymers? J. Rheol. 42 (1998) 1095-1119.

|26| M, Grosso, P.L Maffetone, P. Halin, R. Keunings, V. Legat, Flow of nematic polymers in eccentric cylinder geometry: influence of closure approximations, J. Non-Newtonian Fluid Mech, 94 (2000) 119-134.

127| J.K. Suen, R. Nayak, R.C. Armstrong, R.A. Brown, A wavelet-Calerkin method for simulating the Doi model with orientation-dependent rotational diffusivity. J. Non-Newt onian Fluid Mech. 114 (2003) 197-228.

[28] M. Laso, H.C. Ottinger, Calculation of viscoelastic flow using molecular models: the CONNFFESSIT approacli, J. Non-Newtonian Fluid Mecli, 47 (1993) 1-20.

|29| P. Wapperom, R. Keunings, V. Legat, The backward-tracking Lagrangian particle method for trasient viscoelastic flows, J. Non-Newtonian Fluid Mech. 91 (2000) 273-295.

$|30|$ C.C. Hua, J.D. Schieber, Application of kinetic theory models in spatiotemporal flows for polymer solutions, liquid crystals and polymet melts using the CONNFFESSIT approach, Chem. Eng. Sci. 51 (1996) 1473-1485.

[31] R. Keunings, Micro-macromethods for the multiscale simulation of viscoelastic flow using molecular models of kinetic theory, in: D.M. Binding, $\mathrm{K}$. Walters (Eds.), Rheology Reviews, British Soc iety of Rheology, 2004, pp. 67-98.

|32| M. Doi, Rheological properties of rodlike polymers in isotropic and liqu id crystalline phases. Ferroelectrics 30 (1980) 247-254.

$133 \mid$ R.C. Larson, Arrested tumbling in shearing flows of liquid crystal polymers. Macromolecules 23 (1990) 3983-3992.

134| H.C. Ottinger, Stochastic Processes in Polymeric Fluids: Tools and Examples for Developing Simulation Algotithms, Springer, Betlin, 1996.

|35| W. Maier, A. Saupe, Eine einfache molekulare Theorie des nematischen kristallinflussigen Zustandes, Z. Naturforsch. 13a (1958) 564-570.

|36| M. Calderer, M.G. Forest, Q. Wang. Kinetic theories and mesoscopic models for solutions of nonhomogeneous liquid crystal polymers, J. Non-Newtonian Fluid Mecli. 120 (2004) 69-78.

|37| M. Green, R, Brown, R. Armstrong, Rleological phase diagrams for nonhomogeneous flows of rodlike liquid crystalline polymers, J. Non-Newtonian Fluid Mecli. 157 (2009) 34-43.

[38| M. Green, R. Brown. R. Armstrong. Nonhomogeneous shear flow in concentrated liquid-crystalline solutions, Phys. Flu id. 19 (2007), 111702-111702-4.

|39| H. Yu. P. Zlıang. A kineticálıydeodynam ic simulation of microstructure of liquid crystal polymers in plane shear flow. J. Non-Newtonian Fluid Mech. 141 (2007) 116-127.

$|40|$ M. Kröger, P. Ilg, Derivation of Frank-Ericksen elastic coefficients for polydomain nematics from mean-field moleculat theory tor anisotropic patticles, J. Chem. Phys, 127 (2007) 034903.

|41| J. L. Prieto, R. Bermejo, M. Laso, A semi-Lagrangian micro-macro method tor viscoelastic flow calculations, J. Non-Newtonian Fluid Mech. (2009). doì: $10.1016 j \mathrm{j}$.jnnfm.2009.10.004.

142| D. Talay, L Tubaro, Expansion of the global error for numerical scliemes solving stocliastic differential equations, Stoch. Anal. Appl. 8 (1990) 483-509.

$|43|$ C. Carstensen, Reliable and efficient averaging techniques as universal tool for a posterioti finite element ertor control on unstructured grids, Int. J. Numer. Anal. Mod. 3 (2006) 333-347. 